\title{
6
}

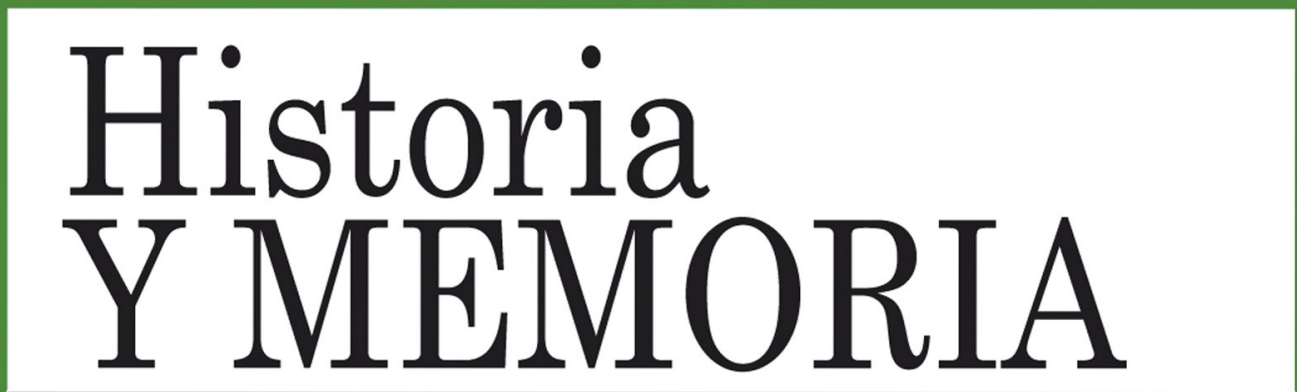

ISSN: 2027-5137

Enero - Junio Año 2013 - Tunja, Colombia

La botica neogranadina de la Compañia de Jesús: un laboratorio para explorar prácticas médicas en la provincia de Santafé, primera mitad del siglo XVIII

Maria Eugenia Osorio Oliveros

Páginas: 143 - 169

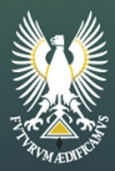




\section{La botica neogranadina de la Compañía de Jesús: un laboratorio para explorar prácticas médicas en la provincia de Santafé, primera mitad del siglo XVIII}

María Eugenia Osorio Oliveros

Universidad de Los Andes-Colombia

Recepción: 15/03/2013

Evaluación: 19/03/2013

Aceptación: 10/04/2013

Artículo de Investigación Científica

\section{Resumen}

Este trabajo se centra en el estudio del inventario realizado en 1767 a la botica que perteneció al Colegio Máximo de Santafé de la Compañía de Jesús. A través del inventario se pretende poner en conocimiento el ejercicio de las prácticas médicas llevadas a cabo por estos misioneros en la provincia santafereña durante la primera mitad del siglo XVIII. Igualmente, se manifiesta la importancia del intercambio de conocimientos de curación entre los jesuitas y los nativos americanos, el cual fue determinante para la instauración colonial y local del paradigma conocido como el galenismo humanista.

Palabras clave: botica Colegio Máximo, medicina jesuita, galenismo humanista, sustancias medicinales americanas. 


\title{
The Nueva Granada pharmacy of the Company of Jesus: a laboratory for the exploration of medical practices in the province of Santafé in the first half of the XVIIIth century.
}

\begin{abstract}
This paper is focused on the study of an inventory carried out in the pharmacy belonging to Colegio Máximo de Santafé of the Company of Jesus in 1767. This inventory is intended to recognize medical practices performed by missionaries in the province of Santafe during the first half of the XVIIIth century. In the same way, importance is given to the exchange of healing knowledge between Jesuits and American natives, which was crucial for the colonial and local establishment of the paradigm of Humanist Galenism.
\end{abstract}

Keywords: Colegio Máximo pharmacy, Jesuit medicine, Humanist Galenism, American medicinal substances.

\section{Introducción}

El protomédico de la ciudad de Santafé, Juan Joseph Cortés, declaró en una carta al señor fiscal el primero de diciembre de 1767 "la importancia de la botica jesuita porque suministraba a la ciudad de los más exquisitos medicamentos y que el cierre de dicha botica afectaba al bien público"; además, entre otras cosas, pretendía administrar el futuro de la botica en ese entonces.

Dicha botica pareció tener un importante impacto en la provincia de Santafé. Si bien el protomédico Cortés estaba detrás de la pertenencia y la administración de este bien exaltando sus virtudes, es común encontrarse reiteradamente hoy en día en algunos estudios sobre 
historia de la medicina colonial la historia de Santafé, y en la historia de la Compañía de Jesús con la fama y el prestigio de esta botica para la ciudad en tiempos coloniales. Por ejemplo, según el historiador Andrés Soriano Lleras en su libro La medicina en el Nuevo Reino de Granada durante la Conquista y la Colonia menciona que: "la botica de la Compañía de Jesús no llegó a cerrarse ni de día ni de noche mientras duró la calamidad". La calamidad hacía referencia a una de las tantas epidemias que eventualmente enfrentó la ciudad en la colonia, siendo los jesuitas quienes se caracterizaron por un papel protagónico en cuanto a la atención de calmar los daños ocasionados por este tipo de enfermedades contagiosas. Sin embargo, al tener en cuenta lo anterior, no dejan de surgir una serie de interrogantes alrededor de la afamada botica: ¿por qué llegaron los jesuitas a ejercer el oficio médico en Santafé? ¿Cuál fue el tipo de medicina practicada en la botica? y ¿cómo llego a configurarse este espacio de la botica santafereña de la Compañía de Jesús en tiempos coloniales?

Para esclarecer tales interrogantes y poder adentrarse a una de las más valiosas boticas de la Nueva Granada, el principal objetivo de esta investigación consiste en dar a conocer la relación que estableció la Compañía de Jesús con las prácticas médicas y farmacéuticas en la provincia de Santafé durante la primera mitad del siglo XVIII. Tal relación se pudo establecer por medio del caso que se presentó con el inventario de los bienes y avalúo de la botica del Colegio Máximo de Santafé realizado a raíz de la expulsión de los jesuitas de los territorios americanos en 1767.

De este modo, este artículo estará separado por dos secciones, las cuales permitirán vislumbrar el escenario colonial de las prácticas médicas de los misioneros en torno a su botica. La primera sección, se enfocará en la descripción del inventario para luego poder reconstruir lo que fue la botica del Colegio Máximo. 
En la segunda sección se explicará el tipo de medicina ejercida por los jesuitas, bajo la premisa que la colonia constituyó la perpetuación y el arraigamiento de las concepciones en torno a lo que llegó a conocerse como el galenismo humanista, sin desconocer a su vez que estas concepciones se vieron acopladas a las propias dinámicas coloniales. Asimismo, con un estudio de la obra del padre Joseph Gumilla, El Orinoco Ilustrado, de 1741 dedicado a la descripción de las plantas de la región de los Llanos Orientales, se pretenderá percibir la construcción de un conocimiento médico por fuera de la botica santafereña, determinando a su vez la importancia de la incorporación y de la utilidad para el desarrollo de las prácticas médicas jesuitas durante la primera mitad del siglo XVIII.

Pero antes de abrir las puertas de la botica jesuita, es preciso tener en cuenta algunas consideraciones historiográficas alrededor del tema que trata la relación de la Compañía de Jesús y la ciencia y su particular desarrollo con las actividades médicas. Estas apreciaciones tienen como fin recalcar la importancia del conocimiento y el aporte de la botica no solo para la historia colonial en Colombia sino también para el estudio de la historia de las ciencias durante este período y en este espacio geográfico.

El tema acerca de la relación entre la Compañía de Jesús y la ciencia en Hispanoamérica ha sido abordado por diferentes autores atómicamente. Podría decirse que este tema no cuenta con una fuerte tradición historiográfica, pero es posible rescatar algunos trabajos cuyo fin es dar a conocer dicho vínculo. En el 2011 el historiador Andrés I. Prieto, de la Universidad de Colorado, publicó un libro titulado: Missionary Scientists: Jesuit Science in the Spanish America 1570-1810 (Misioneros científicos: ciencia jesuita en Hispanoamérica 1570-1810). En su libro, el autor recalca el vínculo que mantuvieron los miembros de esta sociedad entre el desarrollo de sus actividades 
misionales y la ciencia. En ese sentido, Prieto es consciente de examinar dichos estudios desde el propio lente de los misioneros religiosos para, entre otras cosas, establecer diferencias entre los trabajos de los jesuitas en el Nuevo Mundo y aquellos que se encontraban en el Viejo Mundo. El espacio geográfico del trabajo de Prieto es el virreinato de Perú (siglos XVI-XVII) y retoma el análisis de las obras realizadas por los padres José de Acosta, Bernabé Cobo, Niccolo Mascardi, entre otros.

De igual manera, otros trabajos históricos se han interesado por la producción intelectual de la Compañía de Jesús en la colonia, producción que vino acompañada de una serie de textos impresos científicos los cuales abordaron los campos de las matemáticas, la historia natural, la lingüística, y la astronomía. Estos trabajos se caracterizan por manejar herramientas otorgadas por la historia cultural y de las ideas, que a su vez permiten cuestionar conceptos históricos, como por ejemplo el de modernidad y ciencia, y cómo estos se construyeron y fueron asimilados en tiempos coloniales. Para ilustrar lo anterior, es importante considerar el trabajo de la historiadora francesa Antonella Romano, quien lleva una importante trayectoria investigando el vínculo jesuitas-ciencia, especialmente tratando de explicar la configuración y el aporte de una cultura científica producida en el mundo iberoamericano en espacios como colegios, hasta las imprentas que ayudaron a publicar los escritos jesuitas.

Sin embargo, para el caso colombiano el tema aún está por ser explorado en profundidad. Al tratar de encontrar estudios para el contexto de la Nueva Granada, aparecen tres autores a los que es imprescindible acudir al momento de estudiar no solo la historia de los jesuitas en Colombia sino también su relación con el estudio del mundo natural. El primero es el padre Juan Manuel Pacheco S.J. quien en su libro Los Jesuitas en Colombia tomo I-II, tras una extensa recopilación de fuentes primarias intentó abordar todos los puntos de acción ejercidos por la Compañía de 
Jesús. Uno de estos puntos está dedicado a las ciencias y a pesar de que el padre Pacheco no se detuvo a profundizar en este tema, se convierte en un referente esencial para seguir indagando acerca de estas actividades científicas. Adicionalmente, el también padre José del Rey Fajardo, se convierte en otro autorrelevante para comprender la vida intelectual de los jesuitas neogranadinos. La pesquisa del Rey Fajardo es aún más interesante porque en su libro Catedráticos Jesuitas de la Javeriana Colonial (2002), menciona acerca de la labor de los jesuitas boticarios del Colegio Máximo de Santafé, pero desafortunadamente para el autor, y también para el lector, aún este tema es poco estudiado.

En el 2008 fue publicado un libro bajo el nombre de: Scientia Xaveriana. Los Jesuitas y el desarrollo de la ciencia en Colombia (XVI-XIX) del profesor de medicina Alberto Gómez Gutiérrez de la Universidad Javeriana de Bogotá. Acompañado de bellas ilustraciones de manuscritos y de libros antiguos, el libro trata ampliamente de abordar las actividades científicas de los misioneros en distintas regiones de Colombia en un largo período de tiempo. Pero más que ser un libro donde se describen algunas labores de estos miembros en los campos de las ciencias y en donde se esclarecen algunos acontecimientos históricos de la Compañía, el autor hace un llamado de atención e invita a los lectores e investigadores a explorar este campo de estudio.

Otra alternativa para seguir indagando sobre el tema es el camino conducido por la historia de la ciencia en Colombia durante el período colonial. $\mathrm{Al}$ introducirse en este camino atrae la atención la variedad de investigaciones y publicaciones desde el siglo XIX hasta el XXI alrededor de la historia de la medicina y la farmacéutica. Precisamente es en esta línea del desarrollo de las prácticas médicas donde aparecen algunas luces que indican sobre el trabajo de la Compañía de Jesús con las ciencias en la Nueva Granada. 
No obstante solo se tratan de pequeñas luces porque estos trabajos se limitan a referenciar y resaltar la labor asistencial de la Compañía de Jesús al hospital San Juan de Dios en Santafé, o a mencionar la existencia de la valiosa botica santafereña que prestaba sus servicios en tiempos de epidemias tal como se ilustró con la cita de introducción en este texto de Andrés Soriano Lleras. Otros trabajos de autores como el de Pedro María Ibáñez en Crónicas de Bogotá o Datos referentes a la medicina en Santafé de Bogotá de Jorge Tomás Uribe, ayudan a resaltar ambivalencias frente al tema de la botica jesuita. Mientras que en el primer libro Ibáñez menciona que fue esta la primera de la provincia, en el segundo el autor menciona que esta no fue la primera en abrir, pero por carencia en la documentación de fuentes primarias, Tomás Uribe no expande más este caso y deja al lector en el limbo del desconocimiento.

Ahora bien, con respecto a un estudio más avanzado de boticas y de oficios médicos y farmacéuticos cabe subrayar el trabajo realizado por la historiadora bogotana Paula Ronderos. Esta autora se ha dedicado a estudiar y a registrar una historia de boticas en la Nueva Granada. A partir del análisis exhaustivo de los inventarios arrojados en estos espacios, la autora ha descifrado las prácticas médicas, los oficios de los boticarios, los médicos, los cirujanos, los protomédicos, además de revelar el uso y la circulación de objetos, artefactos, tratamientos, recetas médicas que hicieron parte del mundo médico colonial. El trabajo de esta historiadora es un ejemplo de cómo se puede escrudiñar un tema abarcando las condiciones sociales, culturales, políticas sin caer en los abismos del señalamiento histórico; se crea un espacio con una dimensión donde hay dinámicas que logran reflejar una parte de la condición de la sociedad colonial. Igualmente se trae a colación este trabajo porque Ronderos menciona la existencia de los inventarios realizados de las boticas para el caso de Santafé entre los siglos XVII y XVIII, pero no tiene dentro de su lista la botica jesuita, aunque sí tiene 
presente y se apoya en la referencia de un libro del siglo XVI clave para el desarrollo de las prácticas médicas y que perteneció a la librería de botica de la Compañía de Jesús; el libro efectivamente resultó ser inventariado en 1767.

Teniendo en cuenta lo anterior, sigue prevaleciendo la falta de profundización en el tema porque prima más la referencialidad de nombrar hechos o particularidades y no de ahondar o detenerse en explicaciones más rigurosas acerca de las dinámicas ejercidas por los jesuitas tanto en las ciencias como en los oficios de la medicina en el período neogranadino. La motivación de conocer más acerca de la botica de la Compañía de Jesús fue un aliciente que logró encontrar, por ahora, un primer desenlace a partir de los datos proyectados en el inventario de los bienes y avalúo del Colegio Máximo de Santafé en 1767.

\section{El inventario de $\mathbf{1 7 6 7}$}

Después de brevemente cristalizar el panorama científico neogranadino de la Compañía de Jesús, es momento de empezar a conocer el desarrollo de las prácticas médicas jesuitas en Santafé a través del caso que se presentó con la aparición del inventario de su botica en 1767, la cual hacia parte de los servicios del Colegio Máximo. Lo interesante de analizar un inventario en el que solo están listados una serie de utensilios, medicinas en latín, libros, entre otras cosas, es que precisamente se convierte en un rompecabezas cuyas piezas terminan por reconstruir el escenario donde los jesuitas confiaron sus conocimientos médicos a la sociedad santafereña actuando, en cierta manera, como misioneros médicos. Sin excepción habrá piezas perdidas, pero se puede hacer una apreciación que va más allá que la simple y común reseña de algunos trabajos de historia de la medicina colonial, al nombrar la importancia de la botica.

El 15 de noviembre de 1767, el médico y cirujano español Jaime Navarro empezó a inventariar todos los 
elementos que se encontraban en este lugar en compañía del escribano Joseph Rojas. Este médico había llegado a Cartagena en 1760 en compañía del gaditano José Celestino Mutis. Y precisamente, dada su experiencia en el oficio, Navarro fue calificado para realizar esta labor.

El inventario tuvo una duración de seis días, lo que implicó el arduo trabajo de inventariar una de las boticas más abastecidas de sustancias y de objetos medicinales en Santafé. Según las descripciones expuestas en el inventario, la botica era una oficina de dos piezas con tres ventanas de hierro, bastidores de vidrieras y rejas de alambre. La primera pieza medía 9 pasos de largo por 8 pasos de ancho (la cual podría considerarse como una rebotica o despacho, por ser la oficina más pequeña). La segunda pieza medía 14 pasos de largo por 8 pasos de ancho. Alrededor de las paredes de esta segunda pieza se encontraban los estantes de madera con los frascos y los vasos que contenían las sustancias medicinales.

La lógica establecida por Jaime Navarro para inventariar fue por orden alfabético. Este inventario comenzó su lista con sustancias definidas por orden de cada letra sin importar su tipo o clasificación medicinal, es decir, si era un aceite, un jarabe, unas pastillas, o un ungüento. Por ejemplo, para nombrar algunos componentes medicinales que empezaron por la letra A se encontraron inventariados: Acetusvini, Alumen Ustus, Absinth, Azul de Prusia, Axungia de León, todos de diferente tipo medicinal. De igual forma para el día 18 de noviembre, Navarro prosiguió con las sustancias definidas por las letras L (Lac. Terre, Lignusbuxi, Limatura Ferri...); después continuó con aquellas por las letras M, N, O, P, Q, R. Para el día 19 ya había terminado de inventariar aquellas por orden de las letras S, T, V y Z.

El 20 de noviembre Navarro dio fin al inventario con la enumeración de los muebles, los utensilios y los libros. Los muebles listados fueron los mostradores y las estanterías de nogal; algunos utensilios fueron cucharones, ollas con 
tenedores de las medicinas, frascos y botes. Entre los artefactos para prepararlas estaban los alambiques, los almireces y los morteros, y entre los libros que daban cuenta de las instrucciones y procedimientos para preparar una receta médica estaban las farmacopeas y/o materia médica. Todos los libros eran textos en francés, español, italiano y latín y con nombres de autores clásicos como Dioscórides y Galeno. Jaime Navarro concluyó con un sumario y avalúo total del inventario; en él confirma que su manera de proceder fue por A.B.C. y que la botica tuvo un valor estimado de 20.141 .5 pesos y 26 maravedís.

$\mathrm{Al}$ tener presente este inventario como fuente, una de las primeras preguntas que surge es: ¿cómo configurar el espacio físico de esta botica? El primer paso para responder a esta pregunta es situarse dentro del Colegio Máximo de la Compañía de Jesús. Sin duda alguna, educar fue uno de los modos de actuar más relevantes dentro de los ministerios que ejercía la Compañía. El acto de enseñar era considerado un especial conducto para propagar sus misiones apostólicas. Las iglesias, las casas y los colegios fueron los espacios para llevar a cabo procesos de aprendizaje y enseñanza; en algunos casos los colegios adquirieron la categoría de colegiouniversidad ampliando más el sentido de la formación intelectual. Para los jesuitas saber y principalmente cultivar el intelecto representó una necesidad y un principio fundamental. También se puede entender en palabras del historiador español Teófanes Egido que "el jesuita aprendía enseñando a los otros". Detrás de esto estaba la idea de crear una dinámica de asistencia social por medio de la educación especialmente dentro del ámbito urbano donde se creaban modelos de buenos ciudadanos y mejor aun si se trataba de crear ciudadanos cristianos. Si bien los colegios recibían a particulares de clases acomodadas, los menos favorecidos también fueron aceptados. De esta forma el colegio como el medio clave para la 
formación de los misioneros, tuvo una importante acogida y asimilación en las principales ciudades del Viejo Mundo; en igual sentido este modelo organizativo de educación fue implantado en las crecientes provincias del Nuevo Mundo a mediados del siglo XVII hasta 1767.

Para 1604 los misioneros de esta compañía que habían sido enviados a visitar los territorios de la Nueva Granada estaban acomodados en las diferentes provincias. Los primeros que llegaron a la provincia de Santafé fueron los padres José Dadey, Juan Bautista Coluccini, Antonio Grossi, los hermanos Diego Loessing y Pedro Pérez (este último arquitecto). En esta ciudad mandaron a edificar las aulas del Colegio Máximo, el Colegio Mayor de San Bartolomé, la edificación de la iglesia de San Ignacio y posteriormente las residencias del barrio de Las Nieves. Según el padre Joseph Cassani, el Colegio se fundó y se abrió oficialmente en 1604 y el principal objetivo de este era crear un espacio para formalizar en sus aulas la enseñanza de las virtudes cristianas. Una de las particularidades de esta edificación fue el alcance que llegó a tener el colegio al enseñar la lengua muisca por medio de rigurosos estudios lingüísticos realizados por el padre José Dadey.

Aunque el padre Cassani no mencionó la adaptación de una botica dentro del Colegio, el padre Juan Bautista Coluccini logró diseñar un mapa de la organización urbana de los misioneros en Santafé. Este mapa bordeó toda una manzana y en él se encuentran representados el Colegio Máximo, el Colegio-seminario de San Bartolomé y la Iglesia de San Ignacio. En el mapa del padre Coluccini (Imagen 1), la botica se encontraba justo en la esquina de la manzana del Colegio Máximo, hoy en día la ubicación está entre la carrera séptima y la calle novena, o calle de la botica. Coluccini nombra respectivamente estas direcciones bajo los nombres de calle real y calle poco frecuentada, a pesar de que el inventario da cuenta de la botica en el siglo XVIII, el mapa de Coluccini refuerza la existencia de ésta desde mediados del siglo XVII. 


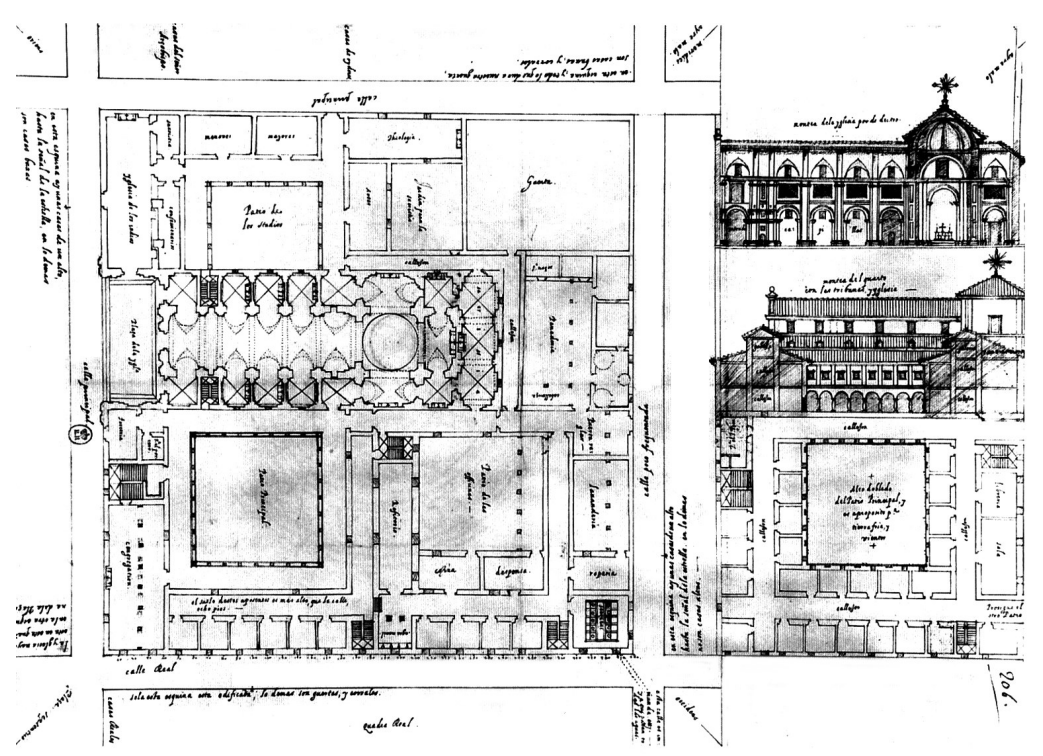

Imagen 1. Mapa realizado por Juan Bautista Coluccini SJ de la organización urbana de la Compañia en Santafé en XVII. El recuadro negro encierra el espacio donde se encontraba la botica. ${ }^{1}$

A partir del inventario de 1767 se pudo concretar un primer paso que era conocer el espacio físico de la botica, respondiendo a las preguntas de dónde se encontraba, cuál fue su dimensión y a partir de cuándo empezó a ofrecer sus servicios. Sin embargo, con respecto al inventario quedaron al final en unos folios una serie de peticiones al señor fiscal que valen la pena ser brevemente expuestos. Estas peticiones -algo ambiguas entre algunos particulares de Santafé- se relacionaron con el ámbito administrativo y el destino final de esta botica.

El médico español Jaime Navarro (encargado de valorar e inventariar el estado de estos bienes

\footnotetext{
${ }^{1}$ La imagen del mapa fue tomado del libro del Museo de Arte Colonial, Desde Roma por Sevilla al Nuevo Reino de Granada: La Compañía de Jesús en tiempos coloniales, (Bogotá: 2004), 41. El museo de Arte Colonial tiene una copia del mapa original el cual reposa en la Biblioteca Nacional de París. En una cronología realizada por el museo de Arte Colonial de una exposición acerca del Colegio Máximo informan que la botica estaba dentro de las instalaciones desde 1663.
} 
pertenecientes a la Compañía), declaró que este proceso presentó dificultades porque muchas medicinas se habían expirado y otras eran anticuadas para la época, lo que le implicó sujetarse a las tarifas más viejas establecidas por el Protomedicato. Con respecto al hermano boticario Bruno Prieto, Navarro mencionó que carecía de todo cuidado en su oficio. ${ }^{2}$ Comprender que algunas de estas sustancias estaban vencidas puede ser lógico. Los miembros de la Compañía dejaron la ciudad a mediados de agosto y el inventario se realizó cuatro meses después en noviembre, ¿cómo no iban a estar en deterioro las medicinas? Lo que sí parece más complejo de entender fue la referencia de Navarro frente algunas sustancias que estaban por fuera de uso y de la práctica médica porque directamente esto conlleva a las preguntas: ¿cuál fue el control ejercido por el protomédico durante el siglo XVIII?, ¿era el protomédico calificado para ejercer control sobre las prácticas en Santafé? y ¿estaba acaso desvaneciendo la autoridad del Protomedicato en el siglo XVIII? Indudablemente para Navarro hubo una ausencia de control por parte de esta institución.

Más intrigante es apreciar la petición de administrar la botica realizada por el protomédico Juan Joseph Cortés quien, como se había mencionado en la introducción, enalteció las virtudes de esta botica para la ciudad. Dentro de su requerimiento Cortés pidió que se le pagaran mil patacones. ${ }^{3}$ Pero el señor fiscal consideró que su solicitud no era pertinente. Esto lo único que manifiesta es oscurecer el panorama porque Cortés, en calidad de protomédico, desde un principio debió haber sido la persona más idónea para inventariar y al parecer su credibilidad fue ignorada inclusive para administrar el futuro de la misma. En contraposición apareció la petición de Antonio Gorraez, quien había sido recomendado por

\footnotetext{
${ }^{2}$ A. H. J. J. M. P., Bogotá, Inventario de los Bienes y Avalúo de la Botica del Colegio Máximo de la Compañía de Jesús 1767. Folios 33-33v.

${ }^{3}$ A. H.J.J.M.P., Bogotá, Inventario de los Bienes y Avalúo de la Botica del Colegio Máximo de la Compañía de Jesús 1767. Folios 35v, 36-36v, 37-37v.
} 
Jaime Navarro para continuar con la administración o por lo contrario, que esta fuera administrada por miembros de la orden Dominica o de la orden Hospitalaria de San Juan de Dios; en ningún momento recomendó al protomédico Cortés. Oficialmente el día 15 de enero de 1768, el señor fiscal de Santafé autorizó a Gorraez y a Navarro a encargarse de los bienes y administración de la botica jesuita.

Finalmente, las anteriores peticiones dejaron entrever un escenario un poco gelatinoso sobre el papel de los funcionarios alrededor de las prácticas médicas a mediados del siglo XVIII, que si bien no hacen parte del tema de este trabajo, por un lado hicieron parte del inventario y por el otro abren las puertas hacia otras investigaciones relacionadas con el campo de historia de la medicina y la administración de la misma. En todo caso estas peticiones recalcan la magnitud y el valor material de la botica que pujaron a resaltar intereses particulares sobre el desenvolvimiento de las prácticas médicas en la colonia.

\section{Una tradición clásica inmersa en la colonia}

Con el inventario de 1767 surgieron otras preguntas que van más allá del plano descriptivo del escenario físico de la botica. Estas preguntas aluden a: ¿cómo explicar las prácticas médicas desarrolladas por los jesuitas en esta provincia?, ¿cuál fue el tipo de medicina utilizada? y ¿cómo los datos arrojados en el inventario se convirtieron en piezas fundamentales para el entendimiento de una asociación entre estas prácticas y los religiosos del Sagrado Corazón de Jesús? Uno de los primeros pasos para dilucidar estas preguntas es comprender que el contexto del ejercicio médico en la colonia estaba relacionado con la reproducción -en las principales provincias neogranadinas- del paradigma clásico conocido como el galenismo humanista, el cual tuvo un mayor predominio durante el siglo XVII logrando mantenerse arraigado al 
escenario colonial prácticamente hasta finales del siglo XVIII.

La tradición occidental del galenismo humanista estaba basada en los estudios desencadenados por la materia médica proveniente del médico griego Hipócrates, la cual permitió desprender una cadena de reinterpretaciones que se perpetuó en el tiempo con los estudios de Galeno (el desarrollo de la teoría humoral hipocrática) los aportes del también griego Dioscórides Anazarbeo (estudio de los reinos de la naturaleza que componen la materia médica y farmacéutica), y los conocimientos de los árabes Alí Abbas, Ibn Ishaq y Avicena. ${ }^{4} \mathrm{El}$ conjunto de estos tratados médicos del mundo antiguo configuraron un paradigma médico el cual se convirtió en el galenismo humanista. Como parte de la dinámica de este corpus médico proporcionado por los antiguos se estudiaron las propiedades curativas de los reinos animal, vegetal y mineral, con el fin de preparar tratados medicinales europeos sobre los procedimientos más adecuados y más útiles. La lógica del paradigma galénico se basó en determinar cómo se debía aplicar un medicamento, con qué se preparaban las sustancias y cuáles eran las enfermedades que el médico debía conocer para ejercer apropiadamente el modelo del galénico. ${ }^{5}$

La importancia de comprender la asimilación de este modelo clásico reviste en la forma como condicionó los ejercicios medicinales tanto en el viejo continente como en el nuevo. El historiador italiano Carlo M. Cipolla mencionó algunas razones para entender la importancia del galenismo humanista. Una de ellas fue la simplicidad, la practicidad y la coherencia con que se podía explicar

\footnotetext{
${ }^{4}$ Pedro Laín Entralgo, Historia Universal de la Medicina, Tomo IV, Medicina Moderna, (Barcelona: Salvat Editores, S.A., 1973) ,182.

${ }^{5}$ Félix Francisco Pastor Frechoso, Boticas, Boticarios y Materia Médica en Valladolid (siglos XVI-XVII), (España: Estudios de Historia de la Ciencia y de la Técnica, Junta de Castilla y de León, Consejería de Cultura y Turismo, 1993), 13.
} 
el modelo para fines medicinales. Nadie en la Europa de la época desde el siglo XVI hasta el XVIII puso en duda dicho modelo porque el mismo contexto en el que se desarrollaron algunas epidemias ayudó a reconfirmar para algunos médicos la certeza y la credibilidad del modelo. Por ejemplo, para algunos médicos europeos fue clara la relación que existía entre los meses calientes y la propagación de las enfermedades, pero no se les pasó por la cabeza, como bien dice Cipolla, que durante estas temporadas de calor había más insectos que propagaron la dispersión de enfermedades. ${ }^{6}$ De modo que este modelo sin ser cuestionado mantuvo una larga duración en la mentalidad médica que reflejó su adaptación en el mundo americano.

Al realizar una clasificación a partir de los tipos de sustancias al inventario de 1767 se pudo descifrar, por ejemplo, las relaciones del uso de estas medicinas con la concepción y la aplicación que se tuvo en la provincia de Santafé del paradigma humoral. Los aceites de vino, manzanilla, albayalde eran emolientes que daban calor al cuerpo. El aceite de traganto era bueno para eliminar las asperezas de la garganta y riñones. El bálsamo de hojas confortaba el estómago. Asimismo, la corteza de alcaparros purgaba las llagas, calmaba los nervios y espasmos. Las enjundias de oso, gallina, tigre calmaban los humores fríos y crasos. ${ }^{7} \mathrm{El}$ zumo de llantén se usaba para calmar las llagas de la boca.

Las anteriores sustancias estaban destinadas a aliviar el cuerpo enfermo bajo los lineamientos regidos por una concepción humoral porque aludían a una

\footnotetext{
${ }^{6}$ Carlo M. Cipolla, Contra un enemigo mortal e invisible, (Barcelona: Editorial Crítica, 1993) 20.

${ }^{7}$ Las enjundias utilizadas en una botica eran gorduras de animales que se mezclaban para componer ungüentos y emplastos que preferiblemente se guardaban en vasos de vidrio. En el inventario de la botica jesuita del Colegio Máximo de Santafé se llegaron a listar enjundias de león (Axungia Leon), de gallina (Axungia gallin), de tigre (Axungia de Tiger) y la enjundia de oso (Axungia Ursi).
} 
parte de la terminología clave del galenismo humanista como calor, purgar, humores fríos, humores crasos. La teoría de los humores o de los fluidos (la sangre, la bilis amarilla, la bilis negra o melancólica y la flema) planteada por el médico griego, consistió en describir que los humores presentan cualidades que se mezclaban entre sí; tales cualidades eran de tipo seco, húmedo, caliente y frío. Cuando había una alteración en alguno de estos humores y sus cualidades, se generaba una reacción desequilibrante ocasionando la aparición de una enfermedad o dolencia corporal. La solución para restaurar el equilibrio corporal se presentaba mediante la aplicación de medicamentos que evacuaría el humor alterado. $^{8}$

Igualmente, una manera para comprender las razones sobre cómo este modelo antiguo galénico alcanzó a ejercer su influencia en las colonias hispanoamericanas, se debió a las políticas instauradas desde el siglo XVI por parte de la corona española. Por una parte, se crearon normas y conductas sobre las prácticas y los ejercicios médicos en las colonias que fueron vigilados por la institución del Real Protomedicato, institución creada en España desde el siglo XVI. Esta institución se encargó de que el modelo clásico fuera ejercido en las colonias hispanoamericanas, y mediante la formulación y la aplicación de leyes no solo pretendió que existiera un orden y una responsabilidad frente a las prácticas médicas sino también trató que los conocimientos médicos fueran proporcionados colectivamente. ${ }^{9}$ Por otra parte, fueron otros los sujetos e instituciones encargados de instaurar las bases del modelo galénico en las colonias, estos fueron los religiosos y los miembros de órdenes religiosas, quienes en una primera instancia encontraron en los recientes

\footnotetext{
${ }^{8}$ Raquel Álvarez Peláez, La Conquista de la Naturaleza Americana, (Madrid: Consejo Superior de Investigaciones Científicas, España, 1993), 342-343.

${ }^{9}$ Paula Ronderos, El dilema de los rótulos. Lectura del inventario de una botica santafereña de comienzos del siglo XVII, (Bogotá: Colección Taller y oficio de la Historia, Pontificia Universidad Javeriana, 2007), 46.
} 
hospitales americanos un mecanismo para difundir la doctrina cristiana.

Particularmente desde finales del siglo XVI (1576) la Compañía de Jesús contó con el respaldo legal de la aparición de una ordenanza apostólica que les permitió ejercer las prácticas de los conocimientos médicos en las colonias hispanoamericanas, exceptuando la cirugía. Los jesuitas aplicaron esta ordenanza en los hospitales, en la prestación de sus servicios de las boticas y en la curación durante sus actividades misioneras. ${ }^{10}$ Precisamente la idea de la asistencia social se convirtió en uno de los ministerios más llamativos entre los jesuitas porque se percibía como un conducto hacia el aprendizaje y la enseñanza apostólica; en otras palabras, la curación debía llevarse desde una dimensión física y espiritual. ${ }^{11}$ La enfermedad en cierto modo ha sido una representación del camino hacia la muerte y los jesuitas se encargaron, antes que nada, de estar al lado del moribundo para instruir en casos terminales el arte del buen morir. ${ }^{12}$ Con respecto al punto anterior, es pertinente refrescar la memoria mencionando algunos casos concretos sobre padres médicos en el Nuevo Reino de Granada. En la ciudad de Cartagena, el padre Alonso Sandoval a finales del siglo XVII prestó sus servicios curativos a los esclavos. En la misma ciudad, el reconocido jesuita

${ }_{10}$ Guillermo Fajardo Ortiz, Los Caminos de la Medicina Colonial en Iberoamérica y las Filipinas, (México: Universidad Nacional Autónoma de México, 1996), 81-82.

${ }^{11}$ Cabe resaltar el trabajo realizado a finales del siglo XVII en Santafé por el padre riobambeño Pedro de Mercado solamente para comprender más la función exegética de los jesuitas en relación con las prácticas médicas. Una versión facsimilar del libro de Mercado, Recetas de Espíritu para enfermos del cuerpo (1680), publicado por Estela Restrepo Zea de la Universidad Nacional en el 2006, permite acercase a la construcción de un tratado para saber curar el alma y saber medicar el cuerpo en momentos de dolencias corporales. También era una forma de fraguar en la mentalidad de los individuos santafereños un orden y una realidad del modelo cristiano por medio de la curación en los momentos más agonizantes de un paciente, la muerte.

${ }^{12}$ Teófanes Egido, y otros, Los jesuitas en España ... 146 
Pedro Claver también atendió a los esclavos enfermos que embarcaron en este puerto y aquellos leprosos que agonizaron en el leprocomio de San Lázaro. ${ }^{13}$ De alguna manera, la aplicación de las concepciones clásicas médicas se amoldaron fácilmente a las actividades pastorales de la comunidad religiosa logrando permear, poco a poco, la continuidad de esta tradición clásica en tierras neogranadinas.

La confluencia entre el carácter científico de la Compañía y el espacio generado por la dinámica colonial, se convirtieron en la perfecta receta para la reproducción del paradigma del galenismo humanista. Fue además en donde se establecieron localmente los hábitos y las percepciones en torno a la curación del cuerpo enfermo. Igualmente el carácter, si se puede decir "abierto", ofrecido por el escenario colonial en torno a las prácticas médicas permitió que existiera una recepción y una introducción por parte de los misioneros jesuitas de los saberes medicinales indígenas, los cuales a su vez se vieron reflejados en la composición del arsenal curativo de la botica jesuita santafereña. Si bien la asimilación dentro del orden colonial neogranadino de las plantas curativas indígenas podría conllevar hacia la investigación y realización de otro artículo, sí es importante en lo posible subrayar y reconocer cómo algunas plantas medicinales habían sido ya incorporadas en el conocimiento médico español desde el siglo XVI y cómo habían sido reproducidos dentro de las prácticas médicas jesuitas en la provincia de Santafé durante las primeras seis décadas del siglo XVIII.

Una pieza fundamental extraída del inventario jesuita fue la sección de libros en la cual el médico Jaime Navarro logró enumerar un total de cuarenta libros. Con base en esta lista, parte del proceso de descifrar e identificar el tipo de medicina aplicada fue más claro

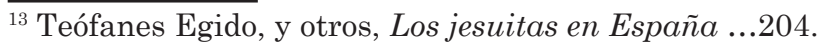


puesto que algunos de los libros ofrecieron detalles para articular la dinámica del escenario interno de la botica. Saber reconocer qué leía el hermano boticario o cómo se suplía de la información necesaria para preparar una receta médica fue indispensable para entender la perpetuación de los ejercicios medicinales. Uno de los libros listados por Navarro fue el del médico sevillano Nicolás Monardes Simplicium Medicamentorum ${ }^{14}$ o comúnmente conocido como Historia medicinal de las cosas que se traen de nuestras Indias Occidentales: ${ }^{15}$ esta fue una de las obras de medicina más traducidas del siglo XVI en el Viejo Mundo. Parte de su reconocimiento correspondió por un lado, a que Nicolás Monardes nunca pisó tierras americanas. Toda su obra representó la descripción de la recolección de datos con las personas que llegaron en las embarcaciones provenientes del Nuevo Mundo al puerto de Sevilla.

Por otra parte, una vez que Monardes estudió el uso medicinal de cada planta, semilla o raíz americana exportada, él las clasificó bajo el paradigma galenista de las renombradas autoridades clásicas como Hipócrates, Galeno, Avicena y Dioscórides ${ }^{16}$; al insertar las plantas americanas dentro de la lógica del modelo clásico Monardes estaba garantizando y contribuyendo a la reproducción y la asimilación de una medicina americana galénica tanto en el Imperio español como en las colonias hispanoamericanas. Algunas de las plantas medicinales de origen americano que fueron analizadas por el médico sevillano como el anime, la tacamaca, la cañafístula, la pimienta blanca, las hojas de tabaco, el bálsamo de Perú, el sasafrás y la raíz de Mechoacán fueron del mismo

\footnotetext{
${ }^{14}$ La versión completa en latín se llamó Simplicium medicamentorum ex novo orbe delatorum, quorum in medicina usus est, publicada en 1579.

${ }^{15}$ Nicolás Monardes, La Historia Medicinal de las cosas que se traen de nuestras Indias Occidentales (1565-1574), versión facsimilar. Introducción de José María López Piñero, (Madrid Ministerio de Sanidad y Consumo) ${ }^{16}$ José Pardo Tomás, El tesoro natural de América. Colonialismo y ciencia en el siglo XVI, (España: Nivola Ediciones, 2002) 81-82.
} 
modo inventariadas como sustancias que hicieron parte de la alacena médica jesuita. Esto permite distinguir que muchas de las medicinas de origen europeo se vieron poco a poco reemplazadas por las sustancias medicinales naturales americanas, facilitando el cumplimiento de llevar a cabo las tareas que implicaban la necesidad de la asistencia médica en Santafé. ${ }^{17}$ Asimismo cada vez más se puede vislumbrar cómo el ejercicio médico aplicado por esta comunidad religiosa dentro de un contexto colonial estuvo determinado por la influencia del espacio dinámico y particular colonial; no solo se trató de importar un modelo con base en los fundamentos clásicos occidentales y aplicarlo directamente a los enfermos americanos sin ningún tipo de alteración en el modelo, para esto también se trató de percibir la necesidad de aplicar una medicina que mantuviera relación con la apropiación y la utilización de las propiedades de las plantas nativas.

Sobre este último punto, cabe anotar que la Compañía de Jesús estuvo al tanto de la adquisición de estos saberes, es por esta razón que vale la pena resaltar la importancia de la obra El Orinoco Ilustrado. Historia Natural, Civil y Geográfica de este Gran Río del padre Joseph Gumilla publicada en $1741,{ }^{18}$ para entrar a proponer una visualización más amplia del espacio de la botica santafereña mediante la circulación de los saberes indígenas en los Llanos Orientales. El padre Gumilla había

\footnotetext{
${ }^{17}$ Paula Ronderos, El dilema de los rótulos...66.

${ }^{18}$ El padre Joseph Gumilla nació en Cárcer, España. Fue lingüista, filósofo y teólogo de la Compañía de Jesús. Como muchos jesuitas que llegaron para asentarse en el territorio neogranadino, Gumilla se estableció primero en las locaciones del Colegio Máximo de Santafé y posteriormente entre 1731 y 1737 se dedicó a la organización de la misión del Orinoco y este padre no fue el único en hacer pública su trabajo sobre el Orinoco. También cabe destacar los trabajos de los padres Juan Rivero con su Historia de las Misiones de los Llanos del Casanare y de los Ríos Orinoco y Meta, y el padre italiano Felipe Salvador Gilij con La Historia geográfica y natural de la provincia del Orinoco. Para ver más sobre las misiones rurales jesuitas consultar: Manuel M. Marzal, La Utopía Posible. Indios y jesuitas en la América colonial (1549-1767) Tomo I. Brasil, Perú, Paraguay y Nuevo Reino, (Perú: Pontificia Universidad Católica del Perú, Fondo Editorial, 1992) 555.
} 
sido enviado a realizar su labor apostólica en esta región de la Nueva Granada, siendo San Ignacio de Betoyes (en el actual Casanare) su misión más apreciable; la misión le permitió a Gumilla movilizarse a lo largo de los Llanos, para controlar por medio de la evangelización, la región de los indígenas Lolacas y los Anabalis. ${ }^{19}$

Estas misiones de igual manera le permitieron establecer a Gumilla un diálogo con los indígenas que lo acercaron a la descripción de las propiedades medicinales de sus plantas y de sus animales. Durante la cotidianidad que evidenció Gumilla en la vida misional de los Llanos Orientales, el jesuita pudo acercarse al conocimiento de las enfermedades de la región, de cómo estas afectaban físicamente el cuerpo y de su curación por medio del uso de cataplasmas, de aceites y de resinas extraídas de los reinos vegetal y animal. ${ }^{20} \mathrm{El}$ reconocimiento del aporte y la utilidad de los saberes nativos en el Orinoco Ilustrado llegó a contar con el consentimiento del fiscal del Consejo de Indias don José Burrul, a quien le pareció que la obra de este padre resultó pertinente para el desarrollo del campo médico: "de donde podrá sacar mucho fruto el profesor de medicina con la noticia de tantas plantas, bálsamos, piedras y animales y lo más precioso de los reinos minerales, vegetal y animal". ${ }^{21}$ Gumilla ayudó a la contribución de un conocimiento médico el cual hizo parte de una cultura impresa jesuita alrededor de la ciencia. Pero a la larga, alguien podría pensar finalmente en: ¿cuál fue el vínculo entre el padre Gumilla y el inventario de 1767 de la botica santafereña? La respuesta estaría enfocada a entender que el padre se convirtió en un referente y apoyo bibliográfico para descifrar el significado de muchas de las plantas americanas descritas en el

\footnotetext{
19 José del Rey Fajardo, Biblioteca de escritores jesuitas neogranadinos, (Bogotá: Editorial Pontificia Universidad Javeriana, Colección Biblioteca Profesional, 2006), 338.

${ }^{20}$ José Rafael Fortique, Aspectos Médicos en la Obra de Gumilla, (Talleres de Italgrafica, 1971) 12.

${ }^{21}$ José Rafael Fortique, Aspectos Médicos...10.
} 
inventario y cuyas descripciones se encontraron bajo los lineamientos del paradigma del galenismo humanista. A pesar de que las sustancias de procedencia americana no ocuparon un importante porcentaje en los estantes de la botica, sí aparecieron generosamente en el inventario y fue gracias a la estrecha relación que establecieron los jesuitas con los indígenas del territorio neogranadino.

En definitiva, aunque la obra de Gumilla no mantuvo una directa relación con el inventario de 1767, permitió establecer una forma de aproximarse al entendimiento de la incorporación a la materia médica colonial de plantas americanas medicinales durante la primera mitad del siglo XVIII. Las propiedades de estas plantas debieron ser seleccionadas bajo criterios y categorías ofrecidas por el modelo clásico, y poder tener una funcionalidad en el oficio médico durante la época. Además la importancia aquí planteada entre la obra del Orinoco Ilustrado y la producción de un conocimiento médico por parte de los miembros de la Compañía de Jesús reviste en dimensionar posibles redes de circulación sobre los saberes americanos dentro de la Nueva Granada. Sin conocer si las plantas medicinales que Gumilla describió en su obra eran después recolectadas y trasladadas o no a la botica del Colegio Máximo de Santafé, no cabe duda la importancia de mencionar el aporte de esta obra para las prácticas médicas. Un mayor estudio de investigación de fuentes para rastrear redes de contacto entre los misioneros y las comunidades indígenas permitiría comprender la configuración local de la medicina establecida por los miembros a los largo de los territorios neogranadinos. Una suposición histórica es proponer que la obtención de plantas americanas a la botica probablemente procedía de actividades misionales que se desplegaron durante la colonia en la Nueva Granada. 


\section{Conclusión}

El inventario de 1767 de la botica del Colegio Máximo de la Compañía de Jesús se convirtió en un caso que permitió desentrañar la comprensión de las prácticas médicas ejercidas por estos misioneros en la provincia de Santafé. Este caso vislumbró la botica como un laboratorio urbano en donde los jesuitas que ejercieron su oficio como hermanos boticarios asimilaron el paradigma del galenismo humanista, para poder así aplicarlo cultural y socialmente dentro de las percepciones de los habitantes santafereños frente a las concepciones de unas prácticas médicas relacionadas con la enfermedad, la curación del cuerpo y la muerte. De la misma manera, se pudo apreciar las distintas caras presentadas en el escenario de la medicina colonial con las problemáticas y los enredos administrativos sobre el destino de la botica jesuita. Esto puso en manifiesto una posible negligencia en torno al oficio médico en la provincia que vale la pena seguir indagando para poder dar cuenta de los diversos intereses públicos y privados alrededor de la medicina durante el siglo XVIII. Si bien existió una legislación dedicada a condicionar la medicina colonial, esta distaba mucho del escenario práctico y emergente de la sociedad en la provincia de Santafé.

La inclusión de la obra del padre Gumilla no fue una casualidad, por el contrario, con el aporte de esta obra se pretendió entrever cómo los jesuitas establecieron una red de conocimientos naturales los cuales convergieron en la configuración y en la circulación de una ciencia jesuita a lo largo de su presencia en tierras americanas. ${ }^{22}$ Dicha ciencia estuvo paralelamente desarrollada por la inclusión de los saberes naturales nativos, los cuales se reflejaron como parte de la configuración de la materia médica colonial que fue trasladada a espacios donde

\footnotetext{
${ }^{22}$ Steven Harris, J., "Mapping Jesuit Science: The Role of Travel in the Geography of Knowledge", en The Jesuits, Cultures, Sciences, and the Arts.1540-1773, (Toronto: University of Toronto, 1999) 215-216.
} 
se llevaron a cabo oficios médicos como hospitales, boticas, leprocomios, visitas particulares y actividades misionales.

Finalmente es importante recordar que todavía quedan fragmentos dispersos un poco nebulosos. Conociendo la proximidad y la relación de la asistencia médica que mantuvo el jesuita Pedro Claver con los esclavos en el puerto de Cartagena queda aún por conocer: ¿qué tantos saberes medicinales por parte de estos esclavos fueron compartidos? o ¿cuáles fueron sus percepciones frente a la enfermedad y la curación? Otras boticas neogranadinas pertenecientes a la Compañía de Jesús quedan aún por ser examinadas, tales fueron las del Colegio Máximo de Antioquia, de Cartagena, o de Tunja. Poder dar pista con estos inventarios sería fundamental para establecer dentro de un marco comparativo los oficios médicos jesuitas en el territorio de la Nueva Granada y poder así seguir ampliando la historia que desentraña la relación entre las prácticas médicas y los jesuitas en tiempos coloniales.

\section{Fuentes documentales}

Archivo Histórico Javeriano, Juan Manuel Pacheco (A. H.J.J.M.P) 1207 Inventario de los Bienes y Avalúo de la Botica del Colegio Máximo de la Compañía de Jesús 1767. Bogotá. La copia original del documento reposa en el Archivo Histórico Nacional de Madrid, España, Fondo Clero-Jesuitas, Legajo 955, Doc. N. 3, América, Santafé de Bogotá (Provincia del Nuevo Reino de Granada) Temporalidades 1767-1768.

Inventario del Colegio Máximo de Quito de la Compañía de Jesús y sus Haciendas durante su Secuestro el 20 de Agosto de 1767. Inventario de la Botica: Folios 25-50. Transcripción realizada por el padre Francisco Piñas Rubio S.J., Biblioteca Aurelio Espinosa Pólit, Quito, Ecuador, 2007.

Monardes, Nicolás. La Historia Medicinal de las cosas que se traen de nuestras Indias Occidentales (1565-1574), versión facsimilar. Introducción de José María López Piñero. Madrid: Ministerio de Sanidad y Consumo. 


\section{Bibliografía}

Álvarez Peláez, Raquel. La Conquista de la Naturaleza Americana. Madrid: Consejo Superior de Investigaciones Científicas, 1993.

Cassani, Joseph S.J., Historia de la Provincia de la Compañía de Jesús del Nuevo Reyno de Granada en la América. Caracas: Biblioteca de la Academia de Historia, Fuentes para la Historia Colonial de Venezuela, 1967.

Cipolla, Carlo M. Contra un enemigo mortal e invisible. Barcelona: Editorial Crítica, 1993.

Egido, Teófanes y otros. Los jesuitas en España y en el mundo hispánico. Madrid: Fundación Carolina. Centro de Estudios Hispánicos e Iberoamericanos, 2004.

Fortique, José Rafael. Aspectos Médicos en la Obra de Gumilla. Talleres de Italgráfica, 1971.

Gómez Gutiérrez, Alberto, Scientia Xaveriana. Los Jesuitas y el desarrollo de la ciencia en Colombia. Bogotá: Pontificia Universidad Javeriana, 2008.

Harris, J. Steven. "Mapping Jesuit Science: The Role of Travel in the Geography of Knowledge", en The Jesuits, Cultures, Sciences, and the Arts.1540-1773. University of Toronto, Canadá, 1999.

Ibáñez, Pedro M. Memorias para la Historia de la Medicina en Santafé. Bogotá: Universidad Nacional de Colombia, Centro de Historia de la Medicina, Imprenta Nacional, 1968.

Laín Entralgo, Pedro. Historia Universal de la Medicina, Tomo IV, Medicina Moderna. Barcelona: Salvat Editores, S.A., 1973.

M. Marzal, Manuel. La Utopía Posible. Indios y jesuitas en la América colonial (1549-1767).Tomo I. Brasil, Perú, Paraguay y Nuevo Reino. Perú: Pontificia Universidad Católica del Perú, Fondo Editorial, 1992.

Museo de Arte Colonial. Desde Roma por Sevilla al Nuevo Reino de Granada: La Compañía de Jesús en tiempos coloniales. Bogotá: 2004. 
Ortiz Fajardo, Guillermo. Los caminos de la medicina colonial en Iberoamérica y las Filipinas. México: UNAM, 1996.

Pardo Tomás, José. El tesoro natural de América. Colonialismo y ciencia en el siglo XVI. España: Nivola Ediciones, 2002.

Pastor Frechoso, Félix Francisco. Boticas, Boticarios y Materia Médica en Valladolid (siglos XVI-XVII). España: Estudios de Historia de la Ciencia y de la Técnica, Junta de Castilla y de León, Consejería de Cultura y Turismo, 1993.

Prieto I. Andrés. Missionary Jesuits. Jesuit Science in Spanish América 1570-1810. Nashville, Tennessee: Vanderbilt University, 2011.

Rey Fajardo, José del, S. J. Catedráticos Jesuitas de la Javeriana colonial. Bogotá: CEJA, 2002.

Rey Fajardo, José del, S. J. Biblioteca de escritores jesuitas neogranadinos. Bogotá: Editorial Pontificia Universidad Javeriana, Colección Biblioteca Profesional, 2006.

Ronderos, Paula. El dilema de los rótulos. Lectura del inventario de una botica santafereña de comienzos del siglo XVII. Bogotá: Colección Taller y oficio de la Historia, Pontificia Universidad Javeriana, 2007.

Soriano Lleras, Andrés. La Medicina en el Nuevo Reino de Granada, durante la Conquista y la Colonia. Bogotá: Biblioteca de Historia Nacional, Editorial Kelly, 1972.

Uribe Ángel, Jorge Tomás. "Datos referentes a la medicina en Santafé de Bogotá". Boletín de Historia y Antigüedades, N. 792: Vol. 83, (Enero-Marzo, 1996).

Valdez Aguilar, Rafael. "Los jesuitas y la medicina naturalista: la planta de la jojoba". Revista de la Facultad de Historia Universidad Autónoma de Sinaloa, N. 12, (septiembrediciembre, 1994). http://historia.uasnet.mx/Clio.htm, (Marzo de 2011).

Villamizar, Carlos Vladimir. "La medicina durante la época colonial colombiana: una aproximación historiográfica". Anuario Colombiano de Historia Social y de la Cultura, N. 30 (2003). 